\title{
Design of an Adaptive Super-Twisting Control for the Cart-Pole Inverted Pendulum System
}

\author{
Yusie Rizal ${ }^{1}$, Muhammad Wahyu ${ }^{1}$, Imansyah Noor ${ }^{1}$, Joni Riadi ${ }^{1}$, Feriyadi ${ }^{1}$, Ronny Mantala ${ }^{2}$ \\ ${ }^{1}$ Dept. of Electrical Engineering, Politeknik Negeri Banjarmasin, Jl. Brigjen H. Hasan Basry, Banjarmasin 70123, Indonesia \\ ${ }^{2}$ Dept. of Business Administration, Politeknik Negeri Banjarmasin, Jl. Brigjen H. Hasan Basry, Banjarmasin 70123, Indonesia
}

\section{ARTICLE INFO}

\section{Article history:}

Received March 24, 2021

Revised April 25, 2021

Accepted April 30, 2021

\section{Keywords:}

Inverted pendulum system;

Stabilization control;

Sliding mode control;

Adaptive super-twisting algorithm;

Open dynamic engine simulation;

anyKode Marilou simulation

\begin{abstract}
A cart-pole inverted pendulum system is one of the underactuated systems that has been used in many applications. This research aims to study the design and the effectiveness of the Adaptive Super-Twisting controller to stabilize the system by comparing it with other previous control methods. A stabilization control of the pendulum upright using the Adaptive SuperTwisting algorithm (ASTA), was investigated. The proposed controller was designed based on the decoupling algorithm method to solve the coupled control input in the system model. We then compared the proposed stabilizing controller with first-order sliding mode control (FOSMC) and Super-Twisting algorithm (STA) in Matlab/Simulink simulation and realistic computer simulation. We developed the computer simulation using anyKode Marilou software, which adopted Open-Dynamic Engine (ODE) as a physics engine. In Matlab/Simulink simulation, we considered three different scenarios: a nominal system, a system with uncertainty, and a disturbed system. Meanwhile, in a computer simulation, we only presented the comparison of different controllers' performances for the realized system. Both results showed that the three controllers could stabilize the pendulum upright with $0.1 \mathrm{rad}$ initial angular position around the vertical axis. Under the same conditions, the ASTA and STA controllers had similar performances; they both have less chattering and faster convergence than the FOSMC approach. However, the FOSMC approach had the least energy delivered and smallest errors than the other two approaches.
\end{abstract}

This work is licensed under a Creative Commons Attribution-Share Alike 4.0

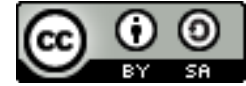

Yusie Rizal

Dept. of Electrical Engineering, Politeknik Negeri Banjarmasin, Jl. Brigjen H. Hasan Basry, Banjarmasin 70123, Indonesia.

Email: yusie.rizal@poliban.ac.id

\section{INTRODUCTION}

The inverted pendulum system is well known as the benchmark for problem-case studies in the control engineering field. Some researchers are attracted to this system because of its characteristics: an unstable, underactuated, and nonlinear system. Thus, some controllers' methods can be designed and tested for such a system plant. This underactuated system has significant applications in real-world engineering problems, for instance, in rockets, underwater robots, walking humanoid robots, overhead cranes, free-flying spacecraft, robotic manipulators, and many more [1]. One practical example of inverted pendulum applications can be found in [2]. Instead of using a complex dynamic model of a certain system, the inverted pendulum model can be employed as a replacement for a simplified model of a longitudinal dynamic of a unicycle robot. The conventional cart-pole inverted pendulum system consists of a cart with an unactuated pendulum attached to the body of the cart. The unactuated pendulum is maintained to stay around its unstable vertical position without falling by controlling the cart's movement. According to [1], an Adaptive Super-Twisting algorithm with decoupled terminal sliding mode control was used to control the inverted pendulum system. In other references, some researchers studied the stabilization control of inverted pendulum system based on output feedback control using Kalman Filter and LQR controller [3], and PID-like fuzzy logic controller [4]. A 
different approach was proposed in [5], where the adaptive control logic was applied to solve the system problem. In general, there are three types of control problems related to inverted pendulum systems: (i) swinging up the pendulum upright from the downward position [6][7][8], (ii) stabilization or balancing of the pendulum upright [7][9][10], and (iii) tracking control of the inverted pendulum [11][12][13][14]. Since this system is popular for several decades, many control methods have been proposed to solve these three problems. This paper investigated the second problem, where the stabilization control of the inverted pendulum upright from its initial position around the equilibrium point.

The Adaptive Super-Twisting control is well-known as robust control and has been employed in many real-world applications. One of the advantages of using adaptive tuning law is the elimination of the requirement of the knowledge regarding upper bounds of external perturbation [1]. Previous researches related to the use of the Adaptive Super-Twisting control method for a cart-pole inverted pendulum system can be found in [1], [6], and [15]. In [1], they use adaptive-super twisting decoupled with the terminal sliding mode control scheme. The system is considered two separate subsystems, and a controller is proposed to steer each subsystem. In contrast to that, we use the coordinate transformation of the decoupling algorithm proposed by Olfati-Saber [16][17] to obtain its normal form. In other research, as presented in [15], they investigate the system with external perturbation and use global sliding mode surface for the system. This means the term of the sliding surface has an exponential term which is a different form of conventional terms for sliding surface. However, in this research, we use the conventional algebraic terms for sliding surface and study the parametric uncertainty (mass uncertainty) and external disturbance for the cart-pole inverted pendulum. Another approach is discussed in [6], they investigate output-feedback controller for a cart-pole inverted pendulum via coupled sliding-mode algorithm. Unlike in [6], we use a full state-feedback controller for such a system via decoupled algorithm to obtain regular form. Moreover, in this work, we improve the previous work as presented in [10] where the controller of LQR, first-order, and second-order sliding mode control was employed. The novelty of this research is the use of an Adaptive Super-Twisting Algorithm controller for a cart-pole inverted pendulum system by utilizing the decoupling algorithm method.

Only conventional first-order and Super-Twisting second-order sliding mode control will be employed as a comparison to the presented controller. These comparisons will be shown in a realistic computer simulation and Matlab/Simulink. In Matlab/Simulink simulation, we provide detailed comparisons to study the control performances for different scenarios, namely, for the nominal system, uncertainty system, and disturbed system, while in a realistic computer simulation, we only present the comparison of controllers for virtual a cart-pole inverted pendulum system without uncertainty and disturbance. We consider this computer simulation because it offers realistic simulated physics [18] in which it adopts Open-Dynamic Engine (ODE). This engine is one of the physics engines in the market even there are other several physics engines such as Bullet physics, NVidea Physics, and DART [19]. The physics engine is the library for simulating rigid bodies in a real-world simulation. Commonly, this computer simulation is intended for robotic systems such as for simulating realistic multi-robot systems [20], modular robots [21], or virtual mechatronics systems. The contribution of this paper is not only the use of the ASTA controller for stabilizing the system plant but also to use the virtual cart-pole inverted pendulum as a testbed for implementing the designed controller. The virtual simulation of the system plant is stable and reliable enough for mimicking this type of underactuated system.

In the realistic simulation presented in this paper, several embedded sensors and actuators are already provided in anykode Marilou, e.g., dc motor, encoder, accelerometer/gyroscope, etc. [22]. Thus, this computer simulation is good enough to imitate and simulate the realizing cart-pole inverted pendulum system. there is an interface program i.e., known as Marilou Open Device Access (MODA) to implement the designed controller in realistic simulation in anyKode Marilou. This interface program can be accessed by $\mathrm{C} / \mathrm{C}++$ programming language using Microsoft Visual $\mathrm{C}++2008$ [22]. Since this work may become an alternative solution to solve the control problem of cart-pole inverted pendulum stabilization in a realistic simulation, then this simulation can also be applied as a low-cost mechatronics system for laboratory testbed in engineering classes. Students and teachers may find this virtual simulation very useful to study control systems and their implementation in robotics and mechatronics system. Moreover, this virtual simulation can also be applied to the research proposed as given in [23] where the proposed teaching robot algorithm is tested in a virtual robot experimentation platform (V-REP). This similar idea can be found in [22][24] for a brain-computer interface to control the wheelchair that is virtually simulated in anyKode Marilou.

\section{RESEARCH METHOD}

\subsection{System Model}

The system model of the cart-pole inverted pendulum is shown in Fig. 1. The dynamic model of this system is given in [25]: 


$$
\begin{aligned}
(m+M) \ddot{y}+m l\left(\ddot{\theta} \cos \theta-\dot{\theta}^{2} \sin \theta\right) & =\tau \\
\ddot{y} \cos \theta+l \ddot{\theta}+g \sin \theta & =0
\end{aligned}
$$

where $m$ and $M$ are the mass of the pendulum and cart, respectively. The $l$ is the length of the pendulum, $\tau$ is the force, $y$ is the displacement, $g$ is the gravitational force, and $\theta$ is the angle of a pendulum to the $\mathrm{z}$-axis. The system (1) can be rewritten as follows:

$$
\begin{aligned}
& \ddot{y}=\frac{\left(l \dot{\theta}^{2}+g \cos \theta\right) m \sin \theta}{M+m \sin ^{2} \theta}+\frac{\tau}{M+m \sin ^{2} \theta} \\
& \ddot{\theta}=-\frac{\left((m+M) g+m l \dot{\theta}^{2} \cos \theta\right) \sin \theta}{M l+m l \sin ^{2} \theta}-\frac{\cos \theta \tau}{M l+m l \sin ^{2} \theta}
\end{aligned}
$$

It is important to note that the system in (2) is the correction for the dynamic model presented by Mahjoub [26][27], in particular, for the negative sign of the first term in the second row of the system (2).

We let $\sin \theta \approx \theta, \cos \theta \approx 1, u=\tau$, and since $\theta$ is around equilibrium point then we assume that $m l \sin ^{2} \theta \approx 0$. We define

$$
\begin{aligned}
& x_{1}=\theta \\
& x_{2}=\dot{\theta} \\
& x_{3}=y \\
& x_{4}=\dot{y}
\end{aligned}
$$

It follows that the state-space in (2) is:

$$
\begin{aligned}
& \dot{x}_{1}=x_{2} \\
& \dot{x}_{2}=-\frac{(m+M) g x_{1}+m l x_{1} x_{2}^{2}}{M l}-\frac{u}{M l} \\
& \dot{x}_{3}=x_{4} \\
& \dot{x}_{4}=\frac{m l x_{1} x_{2}^{2}+m g x_{1}}{M}+\frac{u}{M} \\
& \longrightarrow
\end{aligned}
$$

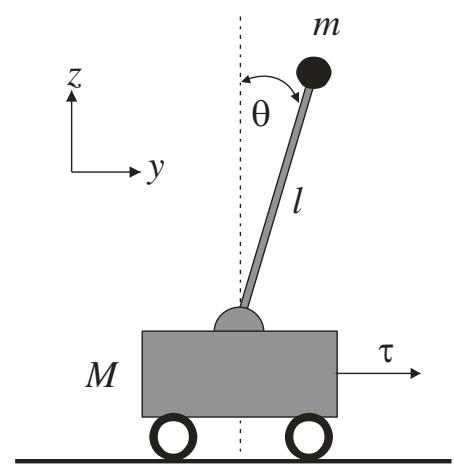

Fig. 1. Cart-pole inverted pendulum model.

\subsection{Decoupling Input}

The control input in the system (4) appears in the second and fourth row. One way to remove the system input in the second row is by considering it as two subsystems as given in [26] and [1]. Another way to solve this problem is to employ the decoupling algorithm proposed by Olfati-Saber [16][17]. This algorithm also applies in different underactuated systems such as wheeled pendular-like systems (WPS) vehicle [28] and inertial wheel inverted pendulum [29]. To decouple these system inputs, we let

$$
\begin{aligned}
& q_{1}=\theta \\
& p_{1}=\dot{\theta}
\end{aligned}
$$




$$
\begin{aligned}
& q_{2}=y \\
& p_{2}=\dot{y}
\end{aligned}
$$

Thus, the equation (5) can be written as:

$$
\begin{aligned}
& \dot{q}_{1}=p_{1} \\
& \dot{p}_{1}=f_{1}(p, q)+g_{1}(p, q) u \\
& \dot{q}_{2}=p_{2} \\
& \dot{p}_{2}=f_{2}(p, q)+g_{2}(p, q) u
\end{aligned}
$$

We use new variables $z_{1}, z_{2}, z_{3}, z_{4}$ and by following global coordinate transformation in [28], we define

$$
\begin{aligned}
& z_{1}=q_{1}-\int_{0}^{q_{2}} \frac{g_{1}(s)}{g_{2}(s)} d s=q_{1}+\frac{q_{2}}{l} \\
& z_{2}=p_{1}-\frac{g_{1}\left(q_{2}\right)}{g_{2}\left(q_{2}\right)} p_{2}=p_{1}+\frac{p_{2}}{l} \\
& z_{3}=q_{2} \\
& z_{4}=p_{2}
\end{aligned}
$$

It follows from (7) that

$$
\begin{aligned}
& \dot{z}_{1}=z_{2} \\
& \dot{z}_{2}=-\frac{g}{l} z_{1}+\frac{g}{l^{2}} z_{3} \\
& \dot{z}_{3}=z_{4} \\
& \dot{z}_{4}=\frac{m g}{M} z_{1}-\frac{m g}{M l} z_{3}+\frac{u}{M}+\Delta
\end{aligned}
$$

Thus, the control input that appears in (4) is removed as in (8). The term $\Delta$ is assumed as disturbance so that it can be ignored in controller design. It is given by:

$$
\Delta=\frac{m l}{M}\left(z_{1}-\frac{z_{3}}{l}\right)\left(z_{2}-\frac{z_{4}}{l}\right)^{2}
$$

\subsection{Controller Design}

In this paper, we assume that all states are available to be measured. We design the sliding surface, $\sigma$ as following [30]:

$$
\sigma=c_{1} z_{1}+c_{2} z_{2}+c_{3} z_{3}+z_{4}
$$

It follows from (10) that

$$
\dot{\sigma}=c_{1} \dot{z}_{1}+c_{2} \dot{z}_{2}+c_{3} \dot{z}_{3}+\dot{z}_{4}
$$

and by substituting ( 8 ) into (11) and ignore the disturbance term of $\Delta$, then we have:

$$
\dot{\sigma}=c_{1} z_{1}+c_{2}\left(-\frac{g}{l} z_{1}+\frac{g}{l^{2}} z_{3}\right)+c_{3} z_{4}+\frac{m g}{M} z_{1}-\frac{m g}{M l} z_{3}+\frac{u}{M}
$$

The system (12) has relative degree 1, and thus, the Super-Twisting Algorithm can be applied. By choosing

$$
u=M\left(u_{e q}+u_{s t}\right)
$$

where

$$
u_{e q}=-\left(\frac{m g}{M}-\frac{c_{2} g}{l}\right) z_{1}-c_{1} z_{2}-\left(\frac{g c_{2}}{l^{2}}-\frac{m g}{M l}\right) z_{3}-c_{3} z_{4}
$$

And $u_{s t}$, the super-twisting algorithm [31][32] is given by:

$$
u_{s t}=u_{1}+u_{2}
$$


where

$$
\begin{aligned}
& u_{1}=-\alpha|\sigma|^{1 / 2} \operatorname{sign}(\sigma) \\
& \dot{u}_{2}= \begin{cases}-u_{s t} & \text { if }\left|u_{s t}\right|>u_{m} \\
-\beta \operatorname{sign}(\sigma) & \text { if }\left|u_{s t}\right| \leq u_{m}\end{cases}
\end{aligned}
$$

and

$$
\operatorname{sign}(\sigma)=\left\{\begin{array}{rr}
1 & \sigma>0 \\
0 & \sigma=0 \\
-1 & \sigma<0
\end{array}\right.
$$

with $u_{m}$ is the upper bound for $u_{s t}$. The control gains $\alpha$ and $\beta$ are adaptively changing by following algorithm [31][33]:

$$
\begin{aligned}
& \dot{\alpha}= \begin{cases}\omega_{1} \sqrt{\frac{\gamma_{1}}{2}} \operatorname{sign}\left(|\sigma|-\mu_{0}\right) & \text { if } \alpha>\alpha_{m} \\
0 & \text { if } \alpha \leq \alpha_{m}\end{cases} \\
& \beta=2 \varepsilon \alpha
\end{aligned}
$$

Where $\omega_{1}, \gamma_{1}, \varepsilon, \mu_{0}$ and $\alpha_{m}$ are nonzero and they need to be tuned to ensure stability. By substituting (13) into (12) using (14)-(15), it follows from (12),

$$
\dot{\sigma}=u_{1}+u_{2}
$$

To stabilize the system, we urge to make the sliding surface $\sigma$ and its derivative $\dot{\sigma}$ converge to zero, i.e.,

$$
\sigma=\dot{\sigma}=0
$$

Hence, if $\sigma \rightarrow 0$ in (10), this implies that $z_{4}, z_{3}, z_{2}$, and $z_{1}$ will also converge to zero.

By following [34], to guarantee $\sigma \rightarrow 0$ in (10), we choose $c_{i}$ for $i=1,2,3$. Let suppose $\sigma=0$ in (10), it follows that

$$
z_{4}=-c_{1} z_{1}-c_{2} z_{2}-c_{3} z_{3}
$$

and by denoting $\mu_{1}=z_{4}$ and $\mu_{2}=\left[\begin{array}{lll}z_{1} & z_{2} & z_{3}\end{array}\right]^{T}$, we may rewrite (20) as

$$
\mu_{1}=c \mu_{2}
$$

where $c=\left[\begin{array}{lll}-c_{1} & -c_{2} & -c_{3}\end{array}\right]$. By taking the first derivative of (10), we have:

$$
\dot{\sigma}=\dot{\mu}_{1}+c \dot{\mu}_{2}
$$

and by relating to (9), it follows that

$$
\dot{\mu}_{2}=\left[\begin{array}{c}
\dot{z}_{1} \\
\dot{z}_{2} \\
\dot{z}_{3}
\end{array}\right]=\left[\begin{array}{c}
z_{2} \\
-\frac{g}{l} z_{1}+\frac{g}{l^{2}} z_{3} \\
z_{4}
\end{array}\right]
$$

i.e., rewritten as:

$$
\dot{\mu}_{2}=A_{1} \mu_{1}+A_{2} \mu_{2}
$$

By substituting (21) into (24), we have

$$
\dot{\mu}_{2}=\left(A_{1} c+A_{2}\right) \mu_{2}
$$

To guarantee $\mu_{2} \rightarrow 0$, we make $\left(A_{1} c+A_{2}\right)$ in (25) to be Hurwitz. Thus, if $\mu_{2} \rightarrow 0$, from (21) we also have $\mu_{1} \rightarrow 0$. Since $\mu_{1} \rightarrow 0$ and $\mu_{2} \rightarrow 0$ then $z_{1} \rightarrow 0, z_{2} \rightarrow 0, z_{3} \rightarrow 0, z_{4} \rightarrow 0$. This implies that $\sigma \rightarrow 0$ is guaranteed. Thus, if this condition is guaranteed, then it is similar to say that by relating (6)-(8) with (10), we have, $y \rightarrow 0, \dot{y} \rightarrow 0, \theta \rightarrow 0$, and $\dot{\theta} \rightarrow 0$.

From the controller design in (13), we have the control law as follow:

$$
\begin{aligned}
u= & M\left[\left(\frac{m}{M}+\frac{c_{2}}{l}\right) g \theta+\frac{\left(m g l-M\left(l^{2} c_{1}+g c_{2}\right)\right)}{M l^{2}} \dot{\theta}+\frac{\left(M c_{2}-m l\right)}{M l^{2}} g y-\frac{\left(c_{1}+c_{3} l\right)}{l} \dot{y}\right] \\
& -\alpha|\sigma|^{\frac{1}{2}} \operatorname{sign}(\sigma)-\int \beta \operatorname{sign}(\sigma) d t
\end{aligned}
$$


Where $c_{1}, c_{2}$, and $c_{3}$ are nonzero. Furthermore, $\alpha$ and $\beta$ are adaptively changing according to (17). Thus, $c_{1}, c_{2}, c_{3}, \omega_{1}, \gamma_{1}, \varepsilon, \mu_{0}$ and $\alpha_{m}$ are needed to be tuned and chosen properly from experiments.

\subsection{Stability Analysis}

To prove the control stability, we use equations from (13)-(16) to substitute $u$ in (12). By calculating (12) for $\dot{\sigma}$, we have

$$
\dot{\sigma}=u_{s t}+\Delta\left(z_{1}, z_{2}, z_{3}, z_{4}\right)
$$

We assume that the disturbance $\Delta\left(z_{1}, z_{2}, z_{3}, z_{4}\right)$ is differentiable and it satisfies the inequality

$$
\left|\dot{\Delta}\left(z_{1}, z_{2}, z_{3}, z_{4}\right)\right|<\delta
$$

where $\delta$ is positive constant. We define the Lyapunov function candidate as

$$
V=\frac{1}{2} \sigma^{T} \sigma
$$

By using $\dot{\sigma}$ in (27) and employing (15)-(16) for switching control $u_{s t}$ and inequality in (28), we compute the first derivative of (29) as following

$$
\begin{aligned}
\dot{V} & =\sigma^{T} \dot{\sigma} \\
& =\sigma^{T}\left(u_{s t}+\Delta\left(z_{1}, z_{2}, z_{3}, z_{4}\right)\right) \\
& =\sigma^{T}\left(-\alpha \sqrt{|\sigma|} \operatorname{sign}(\sigma)-\int \beta \operatorname{sign}(\sigma) d t+\Delta\left(z_{1}, z_{2}, z_{3}, z_{4}\right)\right) \\
& =-\sigma^{T} \alpha \sqrt{|\sigma|} \operatorname{sign}(\sigma)-\sigma^{T} \int \beta \operatorname{sign}(\sigma) d t+\sigma^{T} \Delta\left(z_{1}, z_{2}, z_{3}, z_{4}\right) \\
& \leq-\alpha\left|\sigma^{T}\right| \sqrt{|\sigma|}-\left|\sigma^{T}\right| \int \beta d t+\left|\sigma^{T} \Delta\left(z_{1}, z_{2}, z_{3}, z_{4}\right)\right| \\
& =-\alpha\left|\sigma^{T}\right| \sqrt{|\sigma|}-\left|\sigma^{T}\right| \int \beta d t+\left|\sigma^{T}\right| \int \dot{\Delta}\left(z_{1}, z_{2}, z_{3}, z_{4}\right) d t \\
& =-\alpha\left|\sigma^{T}\right| \sqrt{|\sigma|}-\left|\sigma^{T}\right| \int \beta d t+\left|\sigma^{T}\right| \int \delta d t \\
& =-\alpha\left|\sigma^{T}\right| \sqrt{|\sigma|}-\left|\sigma^{T}\right| \int(\beta-\delta) d t \\
& \leq-\alpha\left|\sigma^{T}\right| \sqrt{|\sigma|}
\end{aligned}
$$

and since $\dot{V}$ is negative semi-definite, then the controller law converges to zero asymptotically [35].

\subsection{Implementation}

To verify the efficacy of the controller design, we conduct two simulations using Matlab/Simulink and ODE-based simulation. The implementation of Matlab/Simulink and ODE simulation can be depicted in the controller's structure as given in Fig. 2 (left), while the realistic simulation of anyKode Marilou is shown in Fig. 2 (right). All state variables of the system are available to measure. In the simulation, we choose the control gains properly, and if the control system is not stable, then the pendulum will fall immediately and vice-versa. To implement the controller laws in ODE simulation for the proposed controller (ASTA), we use the Visual C++ 2008 program to access the virtual inverted pendulum system plant in anyKode Marilou. The IMU (inertial measurement unit) sensor is applied to the realistic simulation to obtain angular position and velocity in the cart-pole inverted pendulum system. This sensor feedback is updated 100 times per second as the control sampling, Ts. The initial position for the pendulum was set up in anyKode Marilou and not from the $\mathrm{C}++$ program. However, in this application program, we may detect this angular position sensor and use it as feedback for the controller. The following algorithm is given and is derived from the controller's structure of ASTA in Fig. 2 (left).

- Initialization:

- Choose the values for $c_{1}, c_{2}$, and $c_{3}$

- Choose values for $\omega_{1}, \gamma_{1}, \varepsilon, \mu_{0}, u_{m}$, and $\alpha_{m}$ 
- Loop:

1. Obtain the values for $\theta, \dot{\theta}, y$, and $\dot{y}$ from sensors' measurements

2. Compute $z_{1}, z_{2}, z_{3}$, and $z_{4}$ in (7)

3. Compute $\sigma$ in (10)

4. Compute $\dot{\alpha}$ and integration of $\alpha$ and $\beta$ in (17)

5. Compute $u_{e q}$ in (14)

6. Compute $u_{1}$ and integration of $u_{2}$ in (16)

7. Compute $u$ in (13)

8. Wait for $T_{s}$ sampling time and then return to step 1

Initially, we choose values for all parameters but only keep $c_{1}, c_{2}$, and $c_{3}$ the same in the two simulation platforms. The remaining parameters are tuned experimentally for the two simulation platforms. In the algorithm program, we cannot calculate the dynamics of the inverted pendulum system in anyKode Marilou because it is a complex system. However, we only can measure its variable states. In Matlab/Simulink, the dynamics model can be calculated using (1) as given in Fig. 2 (left).
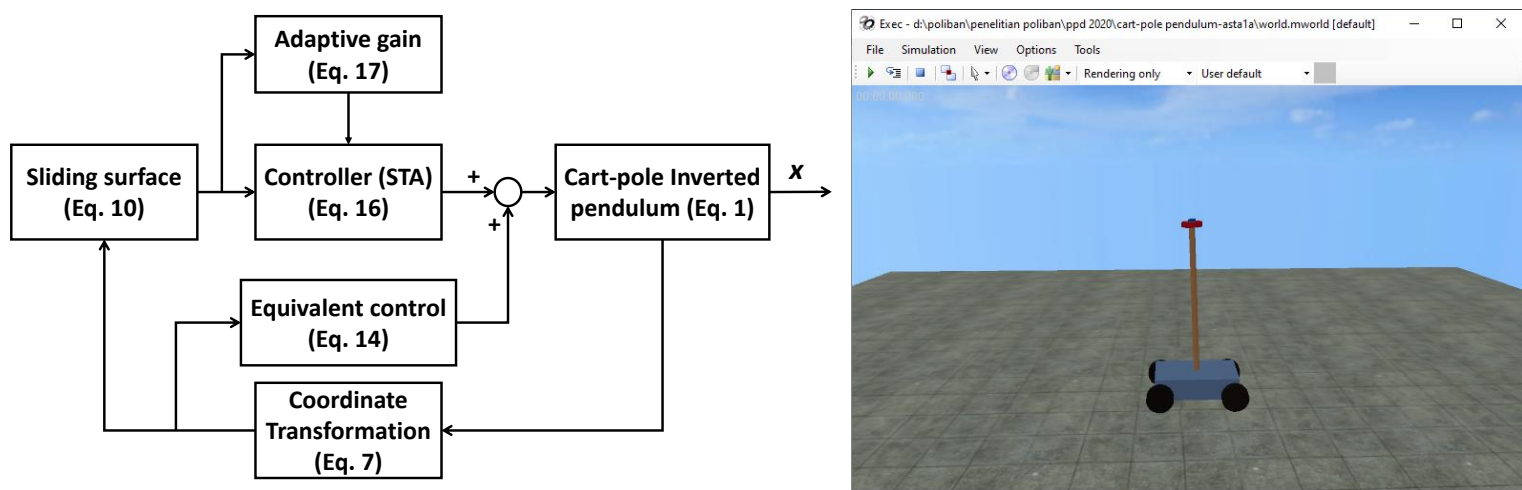

Fig. 2. Structure of ASTA controller (left) and realistic simulation of cart-pole inverted pendulum (right).

\section{RESULT AND DISCUSSION}

This section will show the comparison of the present controller with other stabilizing controller methods (FSOMC and STA). In Matlab/Simulink simulation, we study the performance of controllers in different scenarios, while for anyKode Marilou simulation, we compare the effectiveness of the stabilizing controller for cart-pole inverted pendulum system from these three different control methods. The control gains and sliding surface parameters for each simulation are tuned experimentally. Those values cannot be used directly from one simulation to another simulation because of different platforms. However, the system parameters of the system plant are the same for both simulations, i.e. given by $m=0.25 \mathrm{~kg}, M=1.0 \mathrm{~kg}, l=0.450 \mathrm{~m}$, and $g=$ $9.8 \mathrm{~m} / \mathrm{s}^{2}$. In all simulations, we use the initial angular position for the pendulum with $0.1 \mathrm{rad}$.

\subsection{Matlab/Simulink Simulation}

The control gains for this simulation are $\omega_{1}=1.5, \gamma_{1}=2.25, \varepsilon=1.3, \alpha_{m}=0.25, \mu=0.15, u_{m}=1$, and for sliding surface parameters, we choose $c_{1}=1, c_{2}=3.2$, and $c_{3}=8.2$. We compare the Adaptive Super-Twisting controller with other controllers using FOSMC [34] and STA [36] by conducting three different scenarios for each controllers to stabilize the cart-pole inverted pendulum system, namely, $(a)$ for nominal system, $(b)$ system with uncertainty, and (c) system with disturbance. To compare with other controller methods of FOSMC and STA, we recall from (13) and rewrite:

$$
u=M\left(u_{e q}+u_{s w}\right)
$$

For the FOSMC controller, the switching controller is:

$$
u_{s w}=-h \operatorname{sign}(\sigma)
$$

and for STA, the switching controller is given by [31][32]:

$$
\begin{aligned}
& u_{s w}=u_{1}+u_{2} \\
& u_{1}=-\lambda|\sigma|^{\frac{1}{2}} \operatorname{sign}(\sigma)
\end{aligned}
$$




$$
\dot{u}_{2}= \begin{cases}-u_{s w} & \text { if }\left|u_{s w}\right|>u_{m} \\ -W \operatorname{sign}(\sigma) & \text { if }\left|u_{s w}\right| \leq u_{m}\end{cases}
$$

The control gains for FOSMC is $h=1.36$ and for STA are $\lambda=0.022, W=0.15$, and $u_{m}=1$. The system parameters of $c_{1}, c_{2}$, and $c_{3}$ are kept the same for all controllers (FOSMC, STA, and ASTA). Fig. 3 through Fig. 9 show these simulation results.
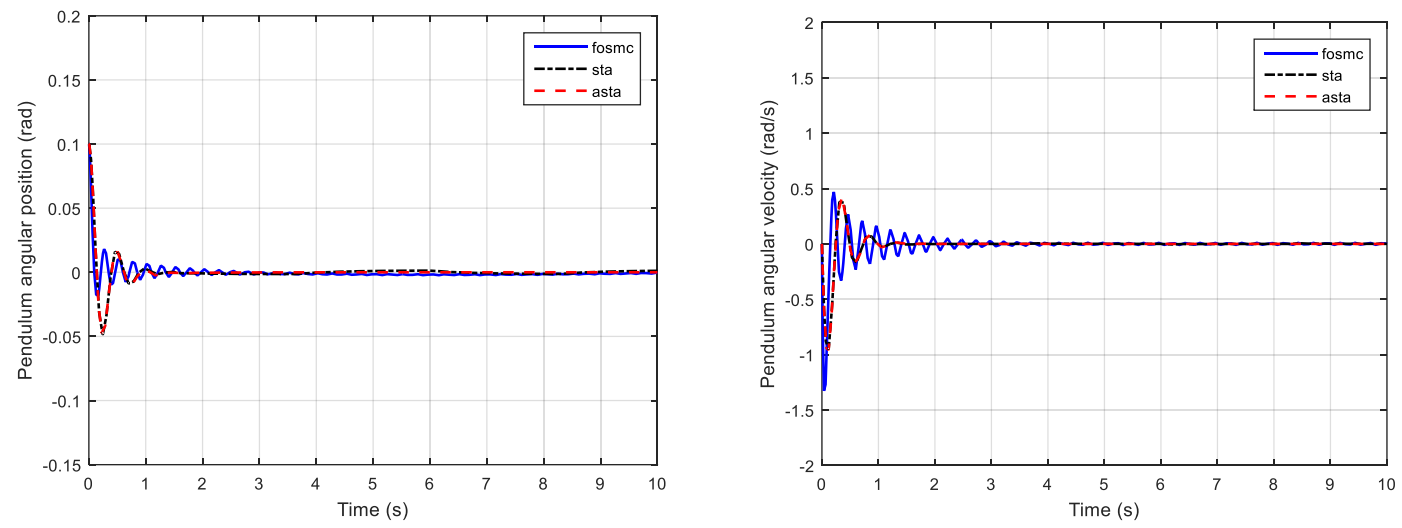

Fig. 3. Angular position (left) and angular velocity of pendulum (right) for system without disturbance.

In Fig. 3, the angular position and velocity of the pendulum for ASTA and STA have the least chattering compared to the FOSMC approach. The angular positions in Fig. 3 (left) for the pendulum using all three controllers can converge to zero in less than 3 seconds. The convergence of angular position and velocity for STA and ASTA methods are similar and better than FOSMC. However, Fig. 4 shows that the system response for the cart's position and velocity of the FOSMC approach has a small error compared to STA and ASTA.
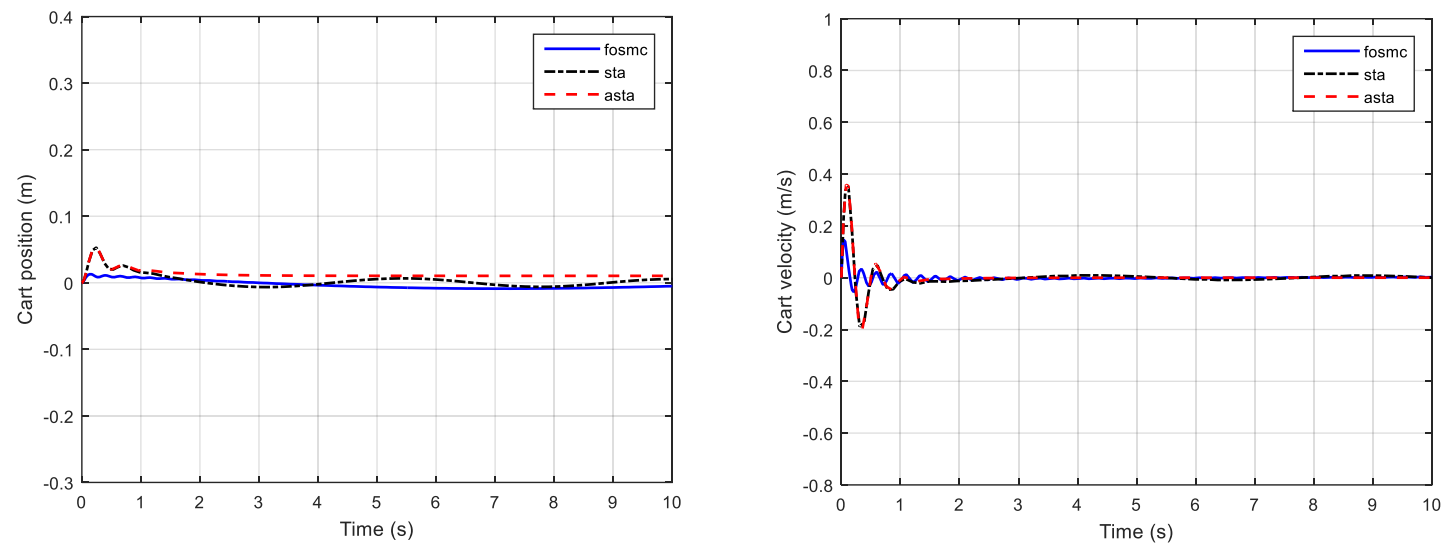

Fig. 4. Position (left) and velocity (right) of the cart for system without disturbance.

Fig. 5 shows the normalized control inputs for nominal system and system with disturbance, respectively. We use normalized control inputs to have fair comparisons between different controllers (FOSMC, STA, and ASTA). In the figure on the right, an impulse signal is injected at 3 sec. All three controllers can overcome this external disturbance. In comparison, we found that the FOSMC approach has more chattering compared to the other two. In the system with uncertainty, the scenario is conducted by changing its masses with $60 \%$ starting from 3 seconds, as shown in Fig. 6 (left). On the right figure, the normalized control input for all three controllers is given. Like the previous scenario, the FOSMC approach has more chattering than STA and ASTA while the responses of STA and ASTA approaches are the same.

The angular positions of the pendulum for disturbance and uncertainty scenarios are in Fig. 7 (left) and (right), respectively. We observed that the angular position for the FOSMC approach is more chattering than the other two controllers. Although the chattering of FOSMC is consistent with previous scenarios, the transient error of this approach is also the same compared with the other controllers. Fig. 8 and Fig. 9 are the response of all states for the three control methods. All state variables $(\theta, \dot{\theta}, y$ and $\dot{y})$ are converging to zeros which implies that the designed controller is correct because it is supported by simulation results. 

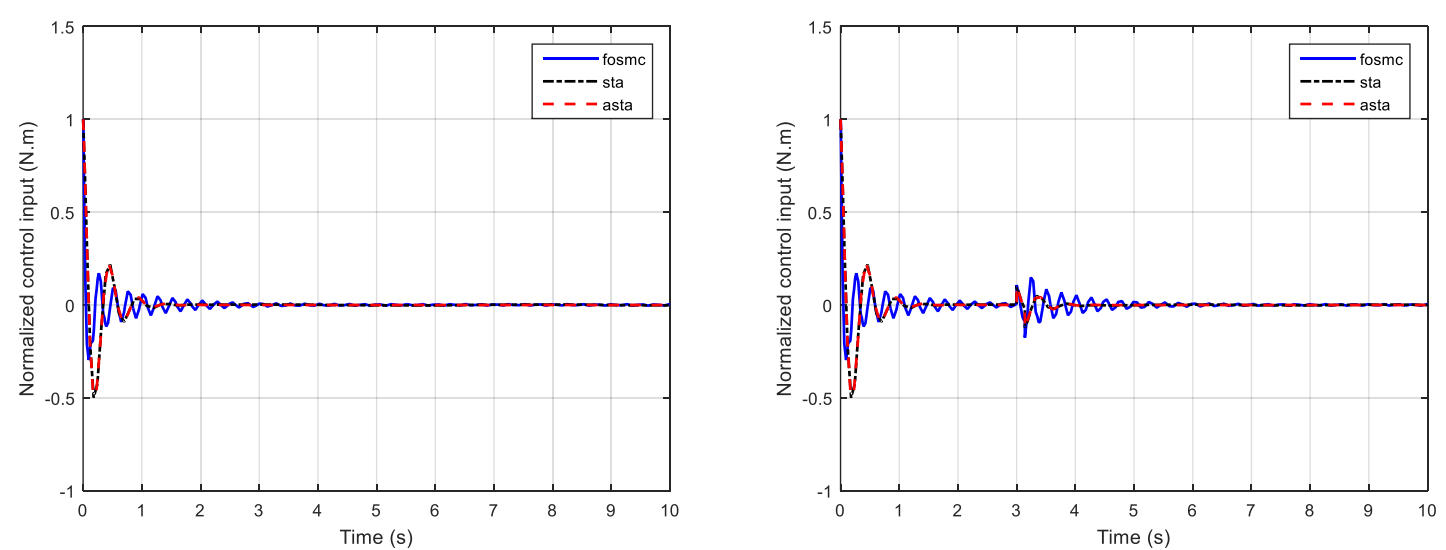

Fig. 5. Control input for system without disturbance (left) and with disturbance (right).
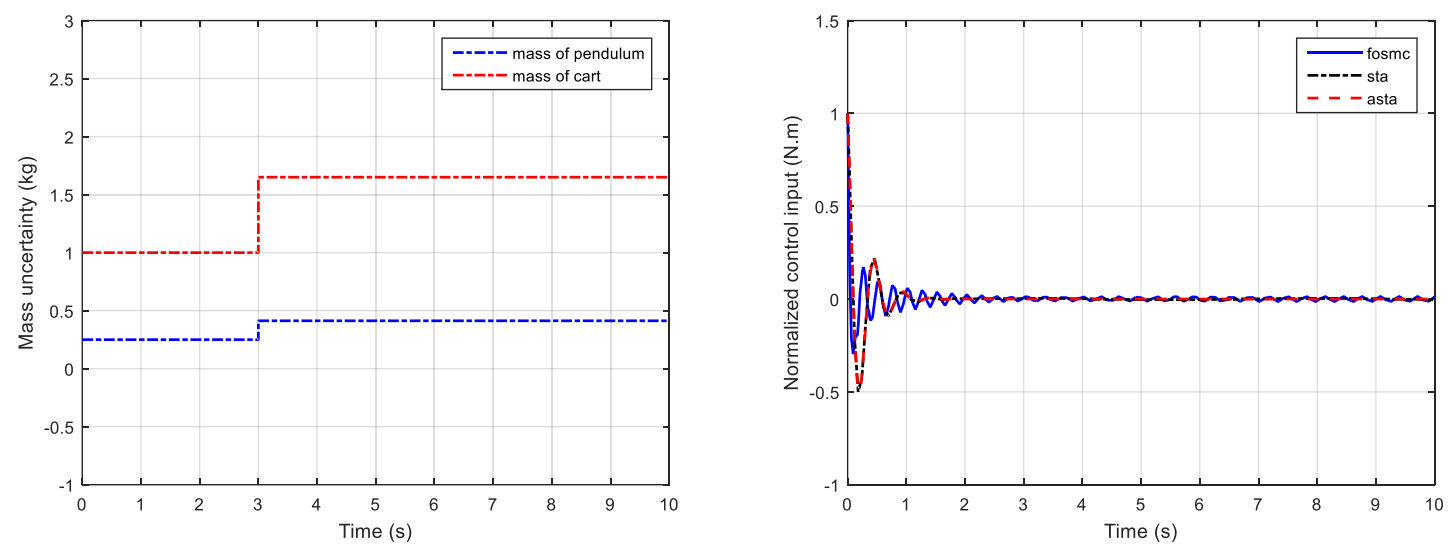

Fig. 6. The system's response for mass uncertainty (left) with its control input (right).
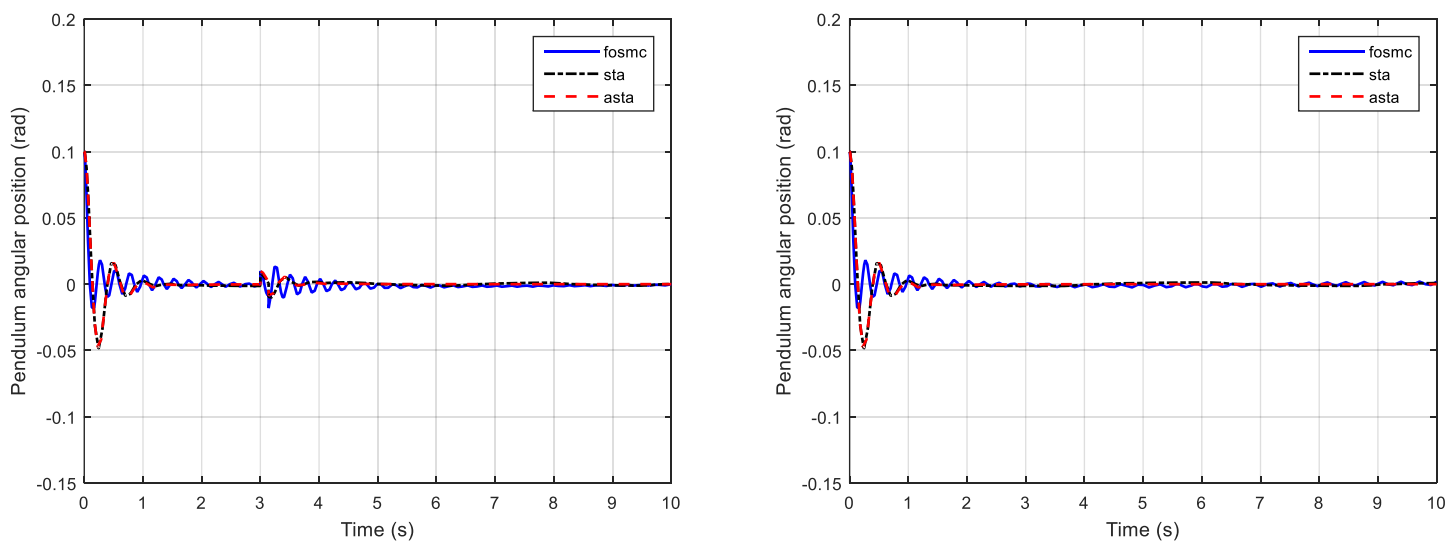

Fig. 7. Angular position of pendulum with disturbance (left) and with uncertainty (right).

The graphical figures of each system and scenario have given system responses, but quantitative comparisons are in Table 1. The performance indexes of each controller in respective scenarios are in Table 1. The integral of the square error (ISE) and integral of absolute error (IAE) using the following formulation:

$$
\begin{aligned}
I S E & =\int e^{2}(t) d t \\
I A E & =\int|e(t)| d t
\end{aligned}
$$


We also provide the energy delivered of the control input using normalized control input as the basis for a fair comparison. The formulation of delivered energy is as follows:

$$
J=\int_{0}^{10} u^{2} d t
$$
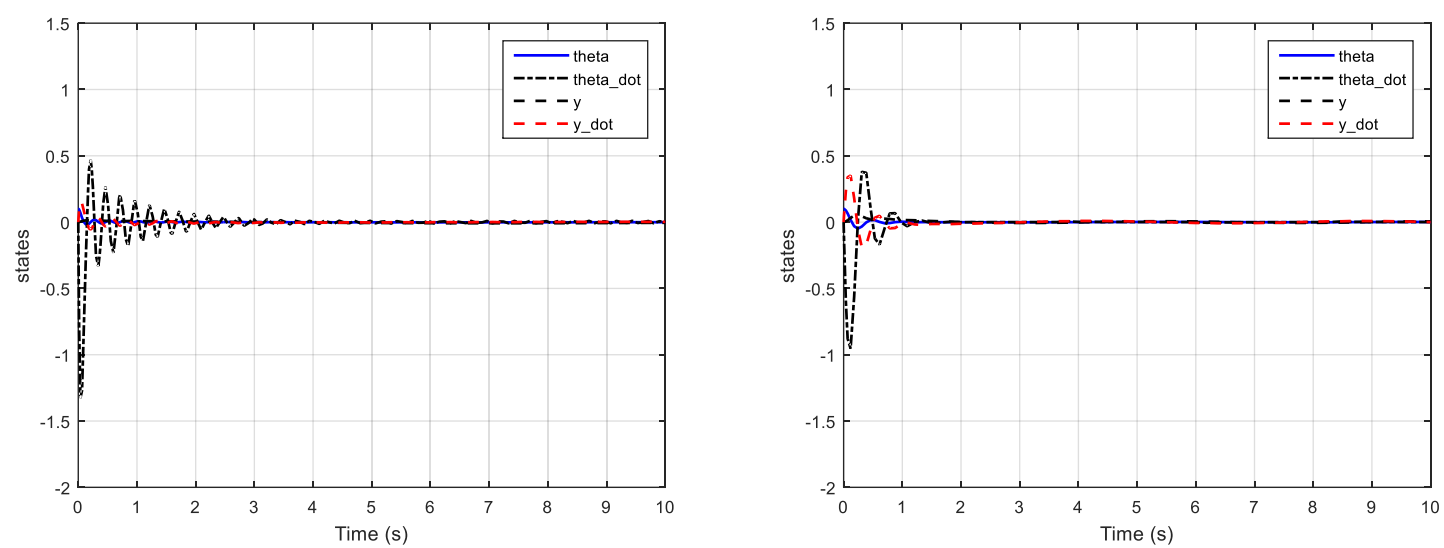

Fig. 8. The system response of FOSMC (left) and STA (right) approaches

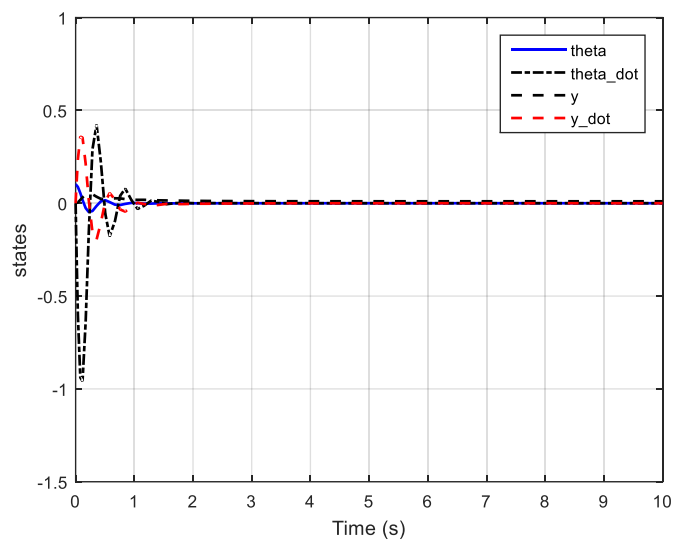

Fig. 9. The system response for ASTA approach.

It is shown from Table 1 that the FOSMC approach has the chattering effect for all scenarios. However, the energy delivered to the system is the least compared to STA or ASTA. In fact, between STA and ASTA approaches, these two controllers have similar delivered energy. This finding is not consistent with the results as given by Mahjoub in [27]. One possible explanation is that the obtained normal form to design controller in this paper is different from Mahjoub [27]. The second reason is the use of dual layers in Mahjoub's work for sliding surfaces. This approach is different from the designed controllers in this paper. Thus, the obtaining results in this paper are different from those presented in [27]. On average, the performance index for integral absolute error and square error for STA and ASTA is twice and 12\% higher than FOSMC, respectively. It implies that the FOSMC method has less steady-state error than the other two methods (STA and ASTA). Since the steady-state error for STA and ASTA are higher than the FOSMC method, this affects the delivered energy to the system in those two methods with 58\% higher than the FOSMC approach. Nevertheless, this would not be the case if the comparison of those three methods control input was not normalized. STA and ASTA methods for disturbance systems are higher than the nominal system with $10.5 \%, 1.62 \%$, and $2 \%$, respectively. But for integral absolute error, we have $17.4 \%, 5.3 \%$, and $16.3 \%$, respectively. On the other hand, the comparison for the uncertainty system for FOSMC and STA controllers tends to less than nominal systems with $1.5 \%$ and $10.34 \%$, respectively. For delivered energy, the system with disturbance for the FOSMC method is $20 \%$ higher than the nominal system. Other comparisons for disturbed systems in STA and ASTA scenarios are only less than $2 \%$ compared to nominal systems. 
Table 1. Comparison of performance indexes and energy delivered for three stabilizing controllers

\begin{tabular}{|c|c|c|c|c|}
\hline \multirow{2}{*}{ Controller } & \multirow{2}{*}{ Scenario } & \multicolumn{2}{|c|}{ Performance Index } & \multirow{2}{*}{$\begin{array}{c}\text { Energy Criterion } \\
\left(\times 10^{2} \mathrm{~J}\right)\end{array}$} \\
\hline & & ISE & IAE & \\
\hline \multirow{3}{*}{ FOSMC [34] } & Nominal & 0.0004706 & 0.02564 & 2.755 \\
\hline & Uncertainty & 0.0004635 & 0.02268 & 2.796 \\
\hline & Disturbance & 0.0005198 & 0.03011 & 3.307 \\
\hline \multirow{3}{*}{ STA [36] } & Nominal & 0.0009681 & 0.02807 & 7.132 \\
\hline & Uncertainty & 0.0008680 & 0.02779 & 7.130 \\
\hline & Disturbance & 0.0009838 & 0.02956 & 7.279 \\
\hline \multirow{3}{*}{ ASTA } & Nominal & 0.0009679 & 0.02148 & 7.122 \\
\hline & Uncertainty & 0.0009679 & 0.02148 & 7.136 \\
\hline & Disturbance & 0.0009865 & 0.02498 & 7.266 \\
\hline
\end{tabular}

\subsection{ODE Simulation}

In this computer simulation, we aim to design a controller that can stabilize the inverted pendulum system. We can visually observe the system response as it is the realization of the system plant. If the controller gains and sliding surface parameters are tuned improperly, then the pendulum is falling. We develop ODE simulation using anyKode Marilou software with a $\mathrm{C}++$ program as a programming interface to access that system plant. In $\mathrm{C}++$ programming, we use the sliding parameters as given, i.e., $c_{1}=0.2526, c_{2}$ $=16.37$, and $c_{3}=6.45$. These three sliding parameters are for all controllers (FOSMC, STA, and ASTA). The control gains for FOSMC is $h=2.325$, and for STA are $\lambda=0.566$, and $\mathrm{W}=0.1025$, while for ASTA are $\alpha=$ $0.522, \omega_{1}=1.70, \gamma_{1}=1.104, \mu=0.025, \alpha_{m}=0.752, \varepsilon=0.25, \mu_{0}=0.027$, and $u_{m}=1$. The sliding parameters and controller gains used in this simulation are different from those in Matlab/Simulink simulation. We tuned all parameters and control gains experimentally.

Fig. 10 shows the comparison of the angular pendulum (left) and normalized control input (right) for three controllers. We observe that the chattering for the FOSMC approach larger than STA and ASTA. These results are consistent with the simulation results in Matlab/Simulink where the system converges in 3 seconds. The comparison of performance indexes and energy delivered to the system using three different controllers is in Table 2.
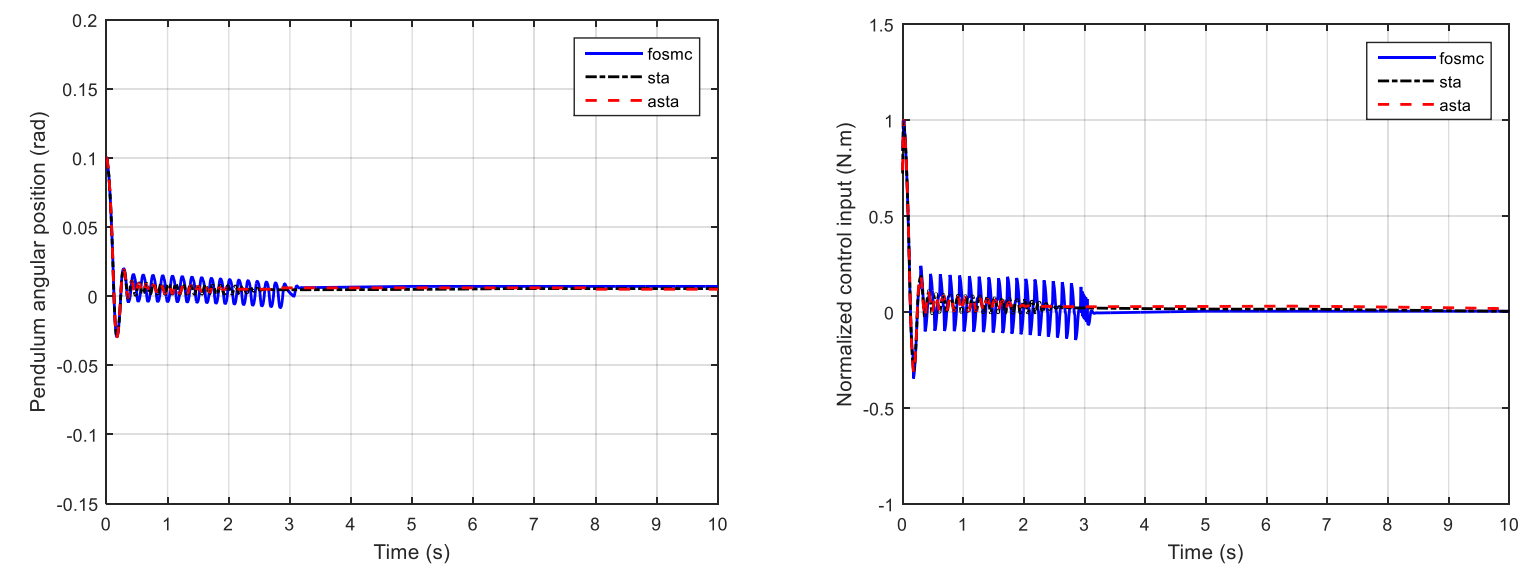

Fig. 10. The system response of angular position of pendulum (left) and normalized control input (right).

In this simulation, we found that the performance indexes in Table 2 for the three controllers are almost the same except for the energy criterion in which FOSMC is slightly larger than STA and ASTA. The comparison of integral square error and absolute error for STA is $23 \%$ and $21.7 \%$, while for ASTA is $15.4 \%$ and $16.2 \%$ smaller than FOSMC, respectively. However, for the energy criterion, the delivered energy using STA and ASTA is $16.2 \%$ and $13.1 \%$ smaller than FOSMC, respectively. These results are different from the simulation results in Matlab/Simulink simulation. From our analysis, this discrepancy is because of different mechanical structures in computed torque control input. In Matlab/Simulink, the simplified model is only for single control input $u$ to actuate the cart, but in ODE simulation, we employ this control input $u$ for driving four wheels of the cart. Thus, from this implementation, we found different results. 
Table 2. Comparison of performance indexes and energy delivered for three stabilizing controllers

\begin{tabular}{|c|c|c|c|}
\hline \multirow{2}{*}{ Controller } & \multicolumn{2}{|c|}{ Performance Index } & \multirow{2}{*}{$\begin{array}{c}\text { Energy Criterion } \\
\left(\times 10^{3} \mathrm{~J}\right)\end{array}$} \\
\hline & ISE & IAE & \\
\hline FOSMC [34] & 0.0013 & 0.0784 & 1.010 \\
\hline STA [36] & 0.0010 & 0.0615 & 0.846 \\
\hline ASTA & 0.0011 & 0.0657 & 0.878 \\
\hline
\end{tabular}

\section{CONCLUSION}

In this work, we proposed the Adaptive Super-Twisting Algorithm as an alternative to solve the stabilizing control problem for the inverted pendulum system. We present the comparison between the proposed controller with existing methods of FOSMC and Super-Twisting Algorithm. To design the controller, we obtain the normal form using the standard decoupling method. Based on this normal form, we design a sliding surface and Adaptive Super-Twisting controller. In simulation results, we provide two different approaches using standard Matlab/Simulink and ODE-based simulations. From these simulations, we found that all three controllers can stabilize the pendulum upright. However, the implementation for each simulation is different, and then, we found some discrepancies between the two simulation results, especially for performance indexes and energy criterion. In graphical figures presented in those two simulations, we observe that the STA and ASTA consistently reduced the chattering effect induced when the FOSMC approach is applied. We found that the use of STA and ASTA for stabilizing such systems is more energyefficient, around 15\% compared to FOSMC. Between the two methods (STA and ASTA), we calculate that the STA method is $3.7 \%$ more efficient than the ASTA method. However, in numerical simulation, those figures are almost similar between STA and ASTA. The findings validated Mahjoub's results [27][26], although the approach used for obtaining the system's normal form is different from those presented in Mahjoub [27][26]. We will continue to work on the same problem for the output-feedback stabilization using the same control method in future work. Unlike the approach in this paper, we assume not all state variables are available to be measured. Thus, this should be more challenging to design the controller and observer.

\section{Acknowledgments}

The authors would like to thank Pusat Penelitian dan Pengabdian Masyarakat Politeknik Negeri Banjarmasin for supporting this research under the scheme of Penelitian Pengembangan Dosen with contract no. 293/PL18/SPK-PG/2020.

\section{REFERENCES}

[1] D. Ashtiani Haghighi and S. Mobayen, "Design of an adaptive super-twisting decoupled terminal sliding mode control scheme for a class of fourth-order systems," ISA Trans., pp. 216-225, 2018. https://doi.org/10.1016/j.isatra.2018.02.006

[2] Y. Rizal, C.-T. Ke, and M.-T. Ho, "Point-to-point motion control of a unicycle robot: Design, implementation, and validation," in IEEE International Conference Robotics and Automation, 2015, pp. 4379-4384. https://doi.org/10.1109/ICRA.2015.7139804

[3] A. Rizky Octavia, D. Nathanlius, and T. Agustinus Tamba, "Implementasi Kontrol Umpan Balik Keluaran Berbasis Tapis Kalman dan Regulator Kuadratik Linier pada Sistem Pendulum Terbalik," J. Otomasi Kontrol dan Instrumentasi, vol. 11, no. 2, pp. 81-93, 2019. https://doi.org/10.5614/joki.2019.11.2.2

[4] A. U. Sambo, F. S. Bala, N. M. Tahir, and A. Y. Babawuro, "Optimal control of inverted pendulum on cart system," J. Phys. Conf. Ser., vol. 1502, no. 1, pp. 1-8, 2020. https://doi.org/10.1088/1742-6596/1502/1/012024

[5] S. Hanwate, Y. V. Hote, and A. Budhraja, "Design and implementation of adaptive control logic for cart-inverted pendulum system," Proc. Inst. Mech. Eng. Part I J. Syst. Control Eng., vol. 233, no. 2, pp. 164-178, 2019. https://doi.org/10.1177/0959651818788148

[6] L. Ovalle, H. Ríos, and M. Llama, "Robust output-feedback control for the cart-pole system: A coupled supertwisting sliding-mode approach," IET Control Theory Appl., vol. 13, no. 2, pp. 269-278, 2019. https://doi.org/10.1049/iet-cta.2018.5249

[7] A. Kukker and R. Sharma, "Genetic Algorithm-Optimized Fuzzy Lyapunov Reinforcement Learning for Nonlinear Systems," Arab. J. Sci. Eng., vol. 45, no. 3, pp. 1629-1638, 2020. https://doi.org/10.1007/s13369-019-04126-9

[8] E. Susanto, A. Surya Wibowo, and E. Ghiffary Rachman, "Fuzzy Swing Up Control and Optimal State Feedback Stabilization for Self-Erecting Inverted Pendulum," IEEE Access, vol. 8, pp. 6496-6504, 2020. https://doi.org/10.1109/ACCESS.2019.2963399

[9] Y. Zheng, X. Li, and L. Xu, "Balance Control for the First-order Inverted Pendulum Based on the Advantage Actor-critic Algorithm," Int. J. Control. Autom. Syst., vol. 18, no. 12, pp. 3093-3100, 2020. https://doi.org/10.1007/s12555-019-0278-z

[10] Y. Rizal, J.-W. Syu, and M.-T. Ho, "Balance control of an inverted pendulum system using second-order sliding 
mode control," in International Automatic Control Conference, 2014, pp. 191-196. https://doi.org/10.1109/CACS.2014.7097186

[11] M. Ashok Kumar and S. Kanthalakshmi, "Hळ tracking control for an inverted pendulum," JVC/Journal Vib. Control, vol. 24, no. 16, pp. 3515-3524, 2018. https://doi.org/10.1177/1077546317750977

[12] M. Ramirez-Neria, Z. Gao, H. Sira-Ramirez, R. Garrido-Moctezuma, and A. Luviano-Juarez, "Trajectory Tracking for an Inverted Pendulum on a Cart: An Active Disturbance Rejection Control Approach," in American Control Conference, 2018, vol. 2018-June, pp. 4881-4886. https://doi.org/10.23919/ACC.2018.8431712

[13] A. K. Jayaprakash, K. B. Kidambi, W. Mackunis, S. V. Drakunov, and M. Reyhanoglu, "Finite-time state estimation for an inverted pendulum under input-multiplicative uncertainty," Robotics, vol. 9, no. 4, pp. 1-26, 2020. https://doi.org/10.3390/robotics9040087

[14] R. G. Guerra, R. Iriarte, J. Eduardo, V. Velázquez, and L. Fridman, "Robust output trajectory linearisation control for a class of linear time-varying systems," IET Control Theory Appl., no. December 2020, pp. 877-889, 2021. https://doi.org/10.1049/cth2.12090

[15] S. Mobayen, "Adaptive global sliding mode control of underactuated systems using a super-twisting scheme: an experimental study," JVC/Journal Vib. Control, vol. 25, no. 16, pp. 2215-2224, 2019. https://doi.org/10.1177/1077546319852257

[16] R. Olfati-Saber, "Global configuration stabilization for the VTOL aircraft with strong input coupling," IEEE Trans. Automat. Contr., vol. 47, no. 11, pp. 1949-1951, 2002. https://doi.org/10.1109/TAC.2002.804457

[17] R. Olfati-saber, "Normal forms for underactuated mechanical systems with symmetry," IEEE Trans. Automat. Contr., vol. 47, no. 2, pp. 305-308, 2002. https://doi.org/10.1109/9.983365

[18] H. L. Pham, B. V. Adorno, V. Perdereau, and P. Fraisse, "Set-point control of robot end-effector pose using dual quaternion feedback," Robot. Comput. Integr. Manuf., vol. 52, pp. 100-110, 2018. https://doi.org/10.1016/j.rcim.2017.11.003

[19] E. Coevoet et al., "Software toolkit for modeling, simulation, and control of soft robots," Adv. Robot., vol. 31, no. 22, pp. 1208-1224, 2017. https://doi.org/10.1080/01691864.2017.1395362

[20] F. M. Noori, D. Portugal, R. P. Rocha, and M. S. Couceiro, "On 3D simulators for multi-robot systems in ROS: MORSE or Gazebo?," in 15th IEEE International Symposium on Safety, Security and Rescue Robotics, Conference, 2017, pp. 19-24. https://doi.org/10.1109/SSRR.2017.8088134

[21] K. J. Gucwa and H. H. Cheng, "RoboSim: a simulation environment for programming virtual robots," Eng. Comput., vol. 34, no. 3, pp. 475-485, 2018. https://doi.org/10.1007/s00366-017-0553-7

[22] Y. Rizal, "Computer Simulation of Human-Robot Collaboration in the Context of Industry Revolution 4.0," in Future of Robotics - Becoming Human with Humanoid or Emotional Intelligence, InTech, 2019. https://doi.org/10.5772/intechopen.88335

[23] Y. Xu, C. Yang, J. Zhong, N. Wang, and L. Zhao, "Robot teaching by teleoperation based on visual interaction and extreme learning machine," Neurocomputing, vol. 275, pp. 2093-2103, 2017. https://doi.org/10.1016/j.neucom.2017.10.034

[24] G. G. Gentiletti, J. G. Gebhart, R. C. Acevedo, O. Yáñez-Suárez, and V. Medina-Bañuelos, "Command of a simulated wheelchair on a virtual environment using a brain-computer interface," IRBM, vol. 30, no. 5-6, pp. 218225, 2009. https://doi.org/10.1016/j.irbm.2009.10.006

[25] J. Zhao and M. W. Spong, "Hybrid control for global stabilization of the cart-pendulum system," Automatica, vol. 37, no. 12, pp. 1941-1951, 2001. https://doi.org/10.1016/S0005-1098(01)00164-9

[26] S. Mahjoub, F. Mnif, and N. Derbel, "Second-order sliding mode control applied to inverted pendulum," in International Conference on Sciences and Techniques of Automatic Control and Computer Engineering, 2013, pp. 269-273. https://doi.org/10.1109/STA.2013.6783142

[27] S. Mahjoub, F. Mnif, and N. Derbel, "Second-order sliding mode approaches for the control of a class of underactuated systems," Int. J. Autom. Comput., vol. 12, no. 2, pp. 134-141, 2015. https://doi.org/10.1007/s11633015-0880-3

[28] M. Yue, C. An, L. Ding, and Y. Zhou, "MPC motion planning-based sliding mode control for underactuated WPS vehicle via Olfati transformation," IET Control Theory Appl., vol. 12, no. 4, pp. 495-503, 2018. https://doi.org/10.1049/iet-cta.2017.0298

[29] A. Hfaiedh, A. Chemori, and A. Abdelkrim, "Disturbance Observer-Based Super-Twisting Control for the Inertia Wheel Inverted Pendulum," in Proceedings of the 17th International Multi-Conference on Systems, Signals and Devices, SSD 2020, 2020, pp. 747-752. https://doi.org/10.1109/SSD49366.2020.9364233

[30] B. Lu, Y. Fang, and N. Sun, "Continuous Sliding Mode Control Strategy for a Class of Nonlinear Underactuated Systems," IEEE Trans. Automat. Contr., vol. 63, no. 10, pp. 3471-3478, 2018. https://doi.org/10.1109/TAC.2018.2794885

[31] S. Rajappa, C. Masone, H. H. Bulthoff, and P. Stegagno, "Adaptive Super Twisting Controller for a quadrotor UAV," in IEEE International Conference on Robotics and Automation, 2016, pp. 2971-2977. https://doi.org/10.1109/ICRA.2016.7487462

[32] M. Derbeli, M. Farhat, O. Barambones, and L. Sbita, "Control of PEM fuel cell power system using sliding mode and super-twisting algorithms," Int. J. Hydrogen Energy, vol. 42, no. 13, pp. 8833-8844, 2017. https://doi.org/10.1016/j.ijhydene.2016.06.103

[33] A. R. Babaei, M. Malekzadeh, and D. Madhkhan, "Adaptive super-twisting sliding mode control of 6-DOF nonlinear and uncertain air vehicle," Aerosp. Sci. Technol., vol. 84, no. September, pp. 361-374, 2019. https://doi.org/10.1016/j.ast.2018.09.013 
[34] J. Liu and J. Liu, "Chapter 9 - Sliding mode control for underactuated system with decoupling algorithm," in Sliding Mode Control Using MATLAB, Academic Press, 2017, pp. 307-327. https://doi.org/10.1016/B978-0-12802575-8.00009-6

[35] Z. Feng and J. Fei, "Design and analysis of adaptive Super- Twisting sliding mode control for a microgyroscope," J. Pone, vol. 13, no. 1, pp. 1-18, 2018. https://doi.org/10.1371/journal.pone.0189457

[36] A. Levant, "Sliding order and sliding accuracy in sliding mode control," Int. J. Control, vol. 58, no. 6, pp. 12471263, 1993. https://doi.org/10.1080/00207179308923053

\section{BIOGRAPHY OF AUTHORS}

Yusie Rizal is a lecturer in Electronics engineering, Politeknik Negeri Banjarmasin, Banjarmasin, Indonesia. Email: yusie.rizal@poliban.ac.id

Muhammad Wahyu is a Lecturer in Electronics engineering, Politeknik Negeri Banjarmasin, Banjarmasin, Indonesia. Email: m.wahyu@ poliban.ac.id

Imansyah Noor is a lecturer in Electronics engineering, Politeknik Negeri Banjarmasin, Banjarmasin, Indonesia. Email: imansyah.poliban@gmail.com

Joni Riadi is a lecturer in Electrical engineering, Politeknik Negeri Banjarmasin, Banjarmasin, Indonesia. Email: joni_riadi@poliban.ac.id

Feriyadi is a lecturer in Electrical engineering, Politeknik Negeri Banjarmasin, Banjarmasin, Indonesia. Email: feriyadi.marzuki@gmail.com

Ronny Mantala is a lecturer in Informatics management, Politeknik Negeri Banjarmasin, Banjarmasin, Indonesia. Email: rmantala@poliban.ac.id 